\title{
Retraction
}

\section{Retracted: Badminton Backcourt Stroke Route Planning Method Based on Deep Learning}

\author{
Journal of Mathematics
}

Received 25 November 2022; Accepted 25 November 2022; Published 25 December 2022

Copyright $\odot 2022$ Journal of Mathematics. This is an open access article distributed under the Creative Commons Attribution License, which permits unrestricted use, distribution, and reproduction in any medium, provided the original work is properly cited.

Journal of Mathematics has retracted the article titled "Badminton Backcourt Stroke Route Planning Method Based on Deep Learning" [1] due to concerns that the peer review process has been compromised.

Following an investigation conducted by the Hindawi Research Integrity team [2], significant concerns were identified with the peer reviewers assigned to this article; the investigation has concluded that the peer review process was compromised. We therefore can no longer trust the peer review process, and the article is being retracted with the agreement of the Chief Editor.

\section{References}

[1] Y. Ma, "Badminton Backcourt Stroke Route Planning Method Based on Deep Learning," Journal of Mathematics, vol. 2021, Article ID 6407049, 6 pages, 2021.

[2] L. Ferguson, "Advancing Research Integrity Collaboratively and with Vigour," 2022, https://www.hindawi.com/post/advancingresearch-integrity-collaboratively-and-vigour/. 


\title{
Badminton Backcourt Stroke Route Planning Method Based on Deep Learning
}

\author{
Yanping Ma \\ Taiyuan University of Technology, Taiyuan 030024, China \\ Correspondence should be addressed to Yanping Ma; mayanping@tyut.edu.cn
}

Received 4 November 2021; Revised 23 November 2021; Accepted 24 November 2021; Published 29 December 2021

Academic Editor: Naeem Jan

Copyright (c) 2021 Yanping Ma. This is an open access article distributed under the Creative Commons Attribution License, which permits unrestricted use, distribution, and reproduction in any medium, provided the original work is properly cited.

\begin{abstract}
In order to improve the planning ability of the badminton backcourt stroke line, this study designs a badminton backcourt stroke line planning method based on deep learning. Firstly, the trajectory adaptive learning method of motion primitives is used to design the hitting line nodes and path space, so as to construct the shortest distributed grid structure model of the hitting line. Then, the constraint parameters of hitting route planning are analyzed, and then the hitting position and player posture are controlled according to node positioning and shortest path optimization deployment. Finally, the adaptive optimization of the route planning process is realized by combining the deep learning method. The simulation results show that this method has good learning control ability and good convergence performance and improves the reliability of badminton backcourt hitting line planning.
\end{abstract}

\section{Introduction}

With the popularization of badminton training, higher requirements are put forward for the efficient training and pertinence of badminton. The key factor for the improvement of effects of badminton training is the badminton backcourt stroke line planning. It is necessary to build an optimized badminton backcourt stroke line planning model, combined with the optimization control method of the badminton backcourt stroke line, adopt artificial intelligence learning algorithm, realize the planning and design of the badminton backcourt stroke line, and improve the stability and reliability of the badminton backcourt stroke, and the design of the relevant badminton backcourt stroke line planning model is of great significance in guiding the optimal training ability of badminton [1].

With the steady improvement of the national economic development level and the continuous progress of the society, badminton is increasingly loved by the masses. Badminton has great sporting advantages. It is not restricted by the site. In addition, it does not require any sports cost investments. Any open place can facilitate people's sports, as long as a pair of rackets and a ball. In addition, badminton is also very helpful to exercise people's endurance and control.
It can also move a variety of joints of the human body, which is very beneficial to enhance physique. However, although badminton has become a popular fitness sport in China and has no sports requirements for ordinary badminton lovers, professional badminton athletes are required to have strong professional skills. They are found through many investigations and practices. The use of multiball training can greatly promote the improvement of badminton players' professional skills [1].

In the traditional methods, the intelligent planning methods for the badminton backcourt stroke line mainly include the badminton backcourt stroke line planning method based on the small-world model [2]. By constructing the grid model of badminton backcourt stroke line planning, combined with spatial grid optimization, the badminton backcourt stroke line planning and design are realized. Based on the method of badminton backcourt stroke line based on ant colony optimization [3], the intelligent planning and design of the badminton backcourt stroke line are based on the node positioning and path optimization control of the badminton backcourt stroke line, and the design is realized through the intelligent planning algorithm. And the badminton backcourt stroke line planning method based on fuzzy PID [4] uses the shortest path optimization and spatial 
parameter estimation, combined with the double programming method, to realize the badminton backcourt stroke line planning. Josue et al. [5] studied the movement pattern of top international male and female badminton players during BMF World Championships. Hung et al. [6] applied the landing strategies in badminton footwork training. Eason et al. [7] analyzed the effects of proactive interference on the learning of backhand strokes.

However, the adaptive optimization ability of the above traditional methods for badminton backcourt stroke line planning is not good, and the spatial positioning ability is not strong. Therefore, aiming at the above problems, this paper proposes a method based on deep learning for the planning of the badminton backcourt stroke route. First of all, the motion primitive trajectory adaptive learning method is used for the planning and designing of badminton backcourt hitting line nodes and path space. Furthermore, the segmented route optimization control method is used for the node positioning and shortest path optimization deployment of the badminton backcourt hitting line. Then, by the use of vertical model measurement and modeling method, the figure model design of the badminton backcourt hitting line is realized. Additionally, the badminton backcourt hitting position and athlete's posture are followed to carry out the linear iterative optimal control. Combined with the deep learning method, the adaptive optimization of badminton backcourt hitting route planning and the motion path planning and design of the target position are apprehended. Finally, the simulation test analysis is carried out which shows the superior performance of the proposed method in improving the ability of badminton backcourt stroke line planning.

\section{Badminton Backcourt Hitting Line Distribution Grid Model and Parameter Analysis}

2.1. Grid Model of Badminton Backcourt Hitting Line Distribution. In order to realize the badminton backcourt stroke line planning based on deep learning, the motion primitive trajectory adaptive learning method is used to plan and design the badminton backcourt stroke line nodes and path space. Firstly, the badminton backcourt stroke line distribution grid model is constructed $[8,9]$, and the location node distribution of the badminton backcourt stroke is obtained, as shown in Figure 1.

According to the location node distribution of the badminton backcourt stroke shown in Figure 1, the shortest path optimization parameter analysis model of badminton backcourt stroke line planning is constructed by using the spatial path-distributed reorganization and optimization control method. According to the geometric description of athletes and the environment [10], the target node optimization of badminton backcourt stroke line planning is carried out [11]; according to the intelligent planning of the badminton backcourt hitting line, the node transmission load is

$$
[\nabla F(x)]_{j}=F(x) \cdot \operatorname{Rot}\left[v_{i}(x)-F(x)\right],
$$

where $F(x)$ represents the objective function of badminton backcourt stroke line planning and $v_{i}(x)$ is the local optimal parameter set of badminton backcourt stroke line distribution. Using the Newton gradient descent algorithm, the parameter estimation and optimization design of badminton backcourt stroke line intelligent planning are carried out [12]. It is obtained that the distribution of random parameters in the badminton backcourt stroke line configuration space is

$$
\left[\nabla^{2} F(x)\right]_{k j}=\frac{1}{\partial F(x)}-2 \sum_{j=1}[\nabla F(x)]_{j} \cdot v_{i}(x) .
$$

Combined with the random parameter distribution results, the shortest path optimization parameters from the initial node to the target node are analyzed, and the linear programming model of path parameters is obtained:

$$
l\left(v_{v}\right)=\operatorname{Tran}\left(P_{e}-P_{0}\right)+\frac{1}{\left[\nabla^{2} F(x)\right]_{k j}},
$$

where $\operatorname{Tran}\left(P_{e}-P_{0}\right)$ represents the translation amount of the path position of the target node. On the differential manifold, according to the analysis of random parameters in the shape space, the path optimization of badminton backcourt hitting line intelligent planning is carried out, and the path optimization equation is

$$
T(t)=\int_{0}^{\infty} l\left(v_{v}\right) \mathrm{d} v+\operatorname{Tran}\left(P_{e}-P_{0}\right) .
$$

The shortest path optimization method is used to design the adaptive spatial parameters of badminton backcourt stroke line planning, and the optimal solution in the distribution nodes $v_{a}, v_{b}$, and $v_{c}$ of the badminton backcourt stroke line is expressed as

$$
l\left(v_{a}\right)=\left\|v_{a}+v_{b}+v_{c}\right\|^{2}-\sum_{t=1} T(t)-l\left(v_{v}\right) .
$$

The segmented route optimization control method is adopted to design the badminton backcourt hitting line distribution grid model, and the shortest path planning design is adopted to obtain the badminton backcourt hitting line shortest distribution grid structure model, as shown in Figure 2.

To sum up, taking the shortest path as the optimization objective function and using the motion primitive trajectory adaptive learning method, the badminton backcourt hitting line node and path space planning and designing are realized in the grid structure model, so as to improve the ability of route planning.

\subsection{Analysis on Constraint Parameters of Badminton Back-} court Hitting Route Planning. The visual servo moving route optimization control method is adopted to carry out the node positioning and shortest path optimization deployment of the badminton backcourt hitting route. Under the node coordinate system, the constraint parameter 


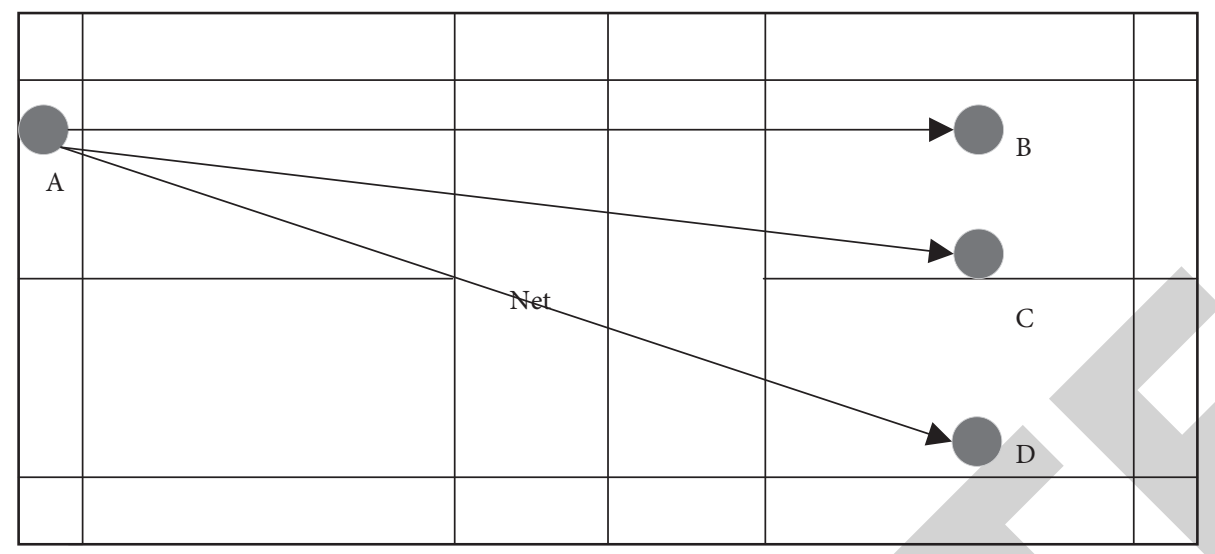

Figure 1: Location node distribution of the badminton backcourt stroke.

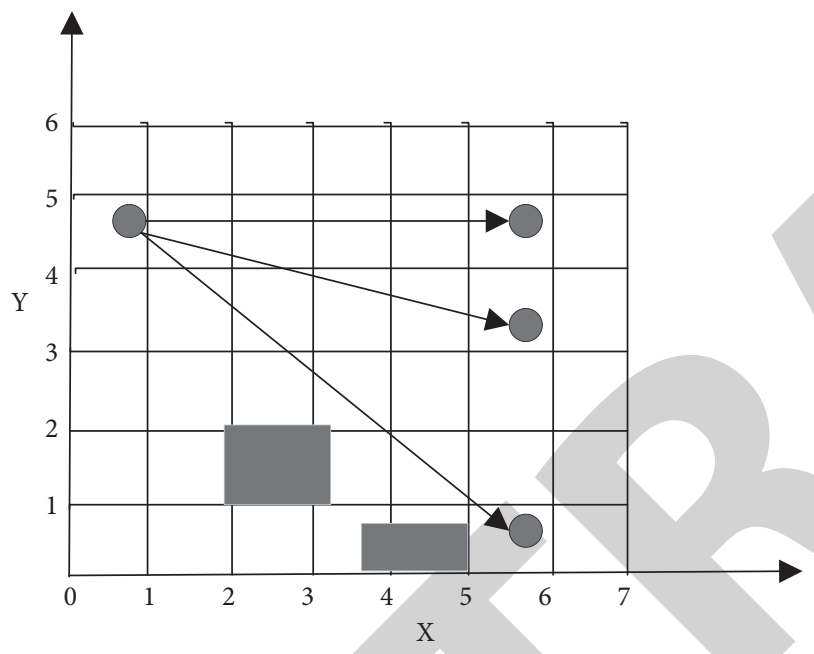

Figure 2: The shortest line distribution grid structure model of the badminton backcourt stroke.

optimization distribution of badminton backcourt hitting route planning is $X_{i}(t)$. In the configuration space starting from the initial posture of badminton backcourt hitting, the line parameter estimation model of badminton backcourt hitting is obtained. Its expression is

$$
\Delta S(t)=\sum_{a-1} l\left(v_{a}\right)+\left(\frac{T_{0}-U_{0}}{X_{i}(t)}\right),
$$

where $T_{0}$ and $U_{0}$, respectively, represent the shortest distribution distance and pose distance of the badminton backcourt hitting line obtained by using the depth camera.

$$
V_{0}=\left\|p^{1}\right\|+\sqrt{T_{6}^{1}+\Delta S(t)}
$$

where $T_{6}^{1}$ represents the deep learning fusion feature distribution set for badminton backcourt stroke line optimization and $p^{1}=\left(p_{x}^{1}, p_{y}^{1}, p_{z}^{1}\right)^{T}$ represents the shortest line fusion parameter of the badminton backcourt stroke line.

Combined with the spatial three-dimensional information sampling method, the depth learning gradient function of badminton backcourt hitting route planning is

$$
\operatorname{Rr}=\sqrt{\Delta T+\Delta U}+\min V_{0},
$$

where $\Delta T$ and $\Delta U$ represent the shortest distribution distance and average position distance of the badminton backcourt hitting line, respectively. Using the method of three-dimensional model measurement and modeling, the figure model design of the badminton backcourt hitting circuit is realized [13]. The characteristic distribution function of the badminton backcourt hitting circuit is $x_{i}, y_{i}$, and $z_{i}$. According to each depth projection corresponding to a programmable badminton backcourt stroke line, the fuzzy control equation of line space planning is obtained:

$$
{ }^{i-1} T_{i}=\frac{\mathrm{Rr}-\sum_{0}^{i} x_{i}+y_{i}+z_{i}}{s+c},
$$

where $s$ represents the positioning error of the badminton backcourt hitting line and $C$ represents the optimization parameters of badminton backcourt hitting line planning.

In the process of badminton backcourt stroke line movement, the starting point of the badminton backcourt stroke line is $P_{0}, P_{1}, P_{2}, \ldots, P_{n}$, and the normal vector of each point is $T$.

According to the above analysis, the optimization parameters of badminton backcourt stroke line planning are obtained, so as to locate the nodes of the badminton backcourt stroke line and deploy the shortest path optimization.

\section{Optimization of the Badminton Backcourt Stroke Route Planning Model}

3.1. Deep Learning of Badminton Backcourt Stroke Route Planning. According to the analysis results of constraint parameters of badminton backcourt stroke line planning, combined with the deep learning method, the intelligent planning and design of the badminton backcourt stroke line are carried out. Given the spatial planning matrix of the badminton backcourt stroke line [14], according to the three-dimensional point cloud information of the scene, the error measurement parameter of badminton backcourt stroke line planning is 


$$
l=\frac{x(t)-1}{h(t, u)}+P_{d}
$$

where $x(t)$ represents the three-dimensional point cloud information points of the badminton backcourt scene and $h(t, u)$ represents the evaluation parameters of the line planning response.

On any $M \times N$-dimensional matrix $A$, combined with iterative optimization and moving parameter analysis, the in-depth learning control of badminton backcourt stroke line planning is carried out, and the in-depth learning quadratic programming model of badminton backcourt stroke line planning composed of $\mathrm{N}$ decision variables is obtained, which is expressed as

$$
F(x)=l \cdot \sqrt{A+h(t, u)} .
$$

An adaptive optimization model of the badminton backcourt hitting line is constructed by using the method of double linear programming, which is expressed as

$$
\eta=\sqrt{E(M A)+E(M B)} \cdot \int_{0}^{\infty} F(x) \mathrm{d} x,
$$

where $E(M A)$ represents the fuzzy membership function value of badminton backcourt stroke line planning and $E(M B)$ represents the inner product parameter of the badminton backcourt stroke line orientation vector.

Through experimental analysis, the stability parameters of the badminton backcourt stroke are estimated, and the optimization function of the equilibrium point position is obtained, which is expressed as

$$
K=f^{2}+\frac{\tau}{2} \cdot\left|\eta^{*} t\right|^{2}
$$

where $\tau$ is the location information in the process of badminton backcourt stroke line planning, $f$ is the fuzzy state characteristic quantity of badminton backcourt stroke line distribution, and $t$ is the time interval of badminton backcourt stroke line optimization.

Based on the optimization results of the equilibrium point position, the optimized depth learning planning function is obtained as

$$
E[V A]=|\sqrt{1+K}|+\int_{0}^{\infty} f(t) \mathrm{d} t .
$$

To sum up, the in-depth learning optimization design of badminton backcourt hitting line planning is realized, and on this basis, the optimization control design is carried out.

\subsection{Optimal Control of Badminton Backcourt Stroke Route} Planning. Based on the deep learning spatial parameter fusion model of badminton backcourt stroke line planning, the deviation parameter analysis model of the badminton backcourt stroke line is analyzed, and the entity prediction sequence distribution of badminton backcourt stroke line planning is expressed as

$$
Q_{i}=2 \sqrt{K}+\sum_{A=1}(E[V A]-1)^{2} .
$$

In the process of continuous optimization of the badminton backcourt hitting line, the adaptive learning approximation control function for line optimization is obtained:

$$
L_{i}=\left\|Q_{i}-E\left[V_{A}\right]^{2}\right\|+\frac{1}{2}
$$

Based on the trajectory optimization and parameter fusion method, the characteristic parameters of the badminton backcourt stroke line planning optimization process are described as

$$
y=\frac{\sqrt{Q_{i}-L_{i}}}{2} .
$$

Combined with the results of characteristic parameters, discuss according to the method of trajectory learning and data fusion, and the degree of freedom trajectory of the badminton backcourt hitting line is expressed as

$$
y(t)=[G+1] \cdot y\left(\sqrt{Q_{i}-L_{i}}\right),
$$

where $G$ is the global transfer matrix. Using the two-degreeof-freedom learning model, the depth learning function of the badminton backcourt stroke is

$$
f(x, y)=\int_{0}^{\infty} \frac{y(t)}{\varepsilon(x, y)} \mathrm{d} t+\frac{L_{i}-y}{g(x, y)},
$$

where $g(x, y)$ and $\varepsilon(x, y)$ represent the independent variables and constraint variables of badminton backcourt stroke path optimization control, respectively.

Through the fusion decision, the fuzzy decision coefficient is $\sigma_{n}^{2}$. Under the single degree of freedom, the inertial tracking parameter model of the badminton backcourt hitting path is

$$
g_{i}^{*}=\sum_{i}^{s_{j}} \frac{f(x, y)+g_{i}}{R}+\sigma_{n}^{2},
$$

where $I$ represents the point set of badminton backcourt stroke path distribution, $R$ represents a normative constant, $x$ represents the reliability constraint parameter of badminton backcourt stroke path optimization, and $y$ represents the two-dimensional spatial planning set of the badminton backcourt stroke path. Its calculation formula is

$$
g_{i}=\sqrt{1-\sigma_{n}^{2}} \cdot \frac{1}{R}
$$

It is assumed that the spatial distribution of the badminton backcourt hitting path is $M \times N$. $M$ and $N$ correspond to the length and width of the spatial distribution of the badminton backcourt hitting path, and the optimal control function of badminton backcourt hitting path planning is 


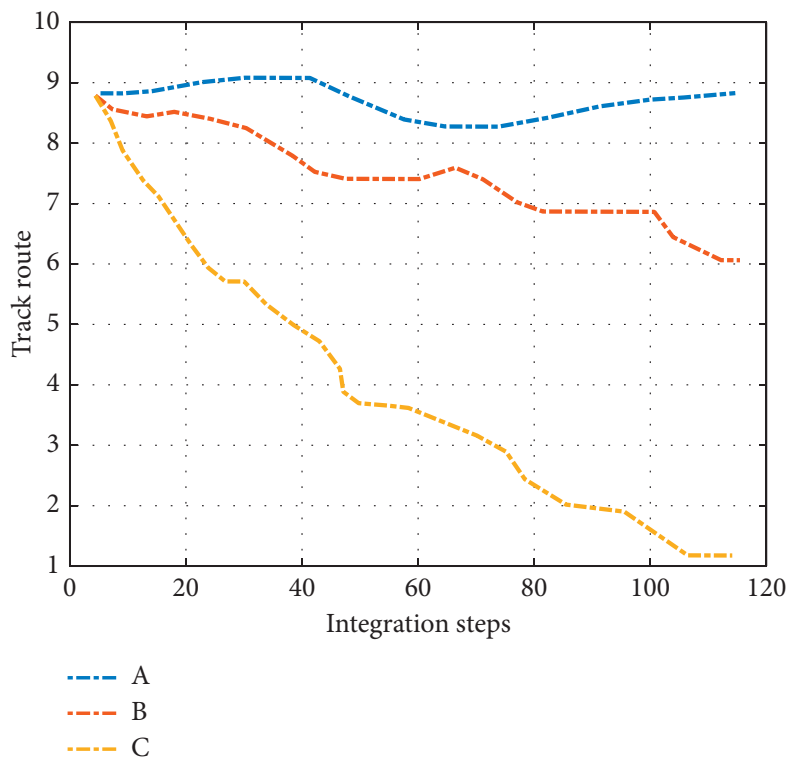

FIgURE 3: Original path of the badminton backcourt stroke.

$$
V\left(M_{m i}\right)=\frac{\sum_{j=1}^{i}(M \times N)}{\Delta u-1}+g_{i}^{*} .
$$

Among them, $\Delta U$ is the gradient parameter of badminton backcourt stroke path planning. Combined with the deep learning method, the optimal path planning control convergence formula is obtained:

$$
F=\frac{p(x, y)+g_{i}}{V\left(M_{m i}\right)}
$$

where $p(x, y)$ represents the convergence parameter set.

To sum up, the linear iterative optimal control is carried out according to the badminton backcourt hitting position and player posture, so as to realize the adaptive optimization of badminton backcourt hitting route planning and the motion path planning and design of the target position.

\section{Simulation Experiment}

In order to test the performance of this method in realizing badminton backcourt stroke path planning, the simulation test is carried out. The parameters are set as that the spatial parameters of the badminton backcourt stroke are $200 * 300$, the number of entities of backcourt stroke path planning is $n=300$, the maximum number of iterations of deep learning is 250 , and the coefficient of adaptive learning is 0.37 . The original path of badminton backcourt hitting in different groups is obtained, as shown in Figure 3.

Taking the original path in Figure 3 as the experimental data, this method is used to optimize the badminton backcourt hitting position and player posture, and the optimized path planning results are shown in Figure 4.

According to the analysis of Figure 4, the route planning and adaptive optimization of the badminton backcourt stroke can be effectively realized by using this method, and the optimization effect is obvious.

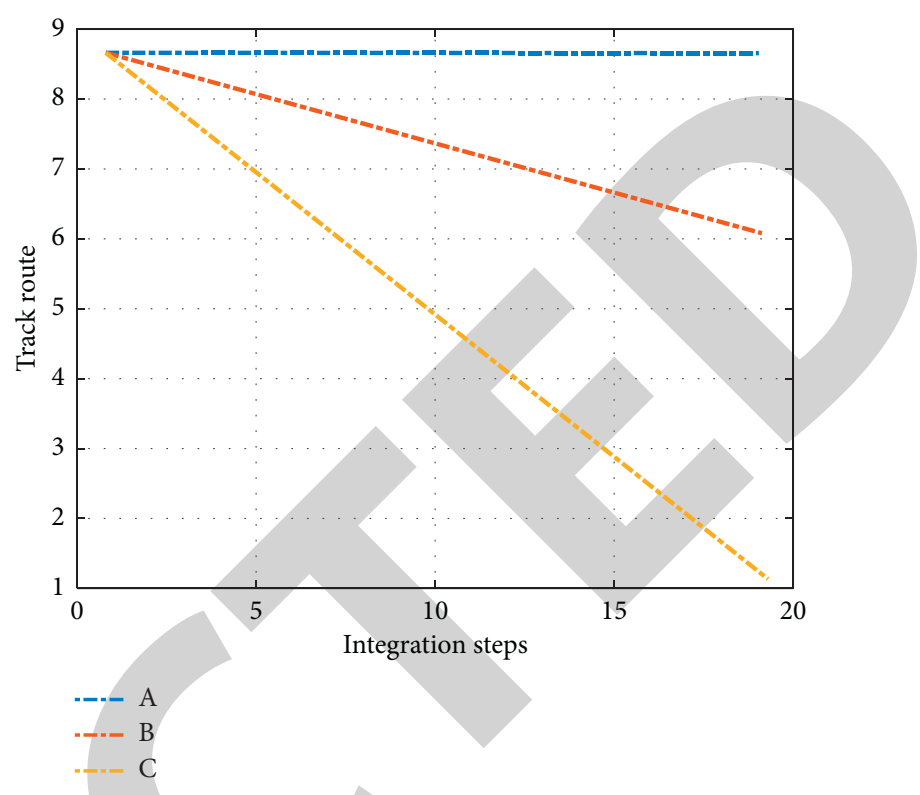

FIGURE 4: Optimized badminton backcourt stroke path planning results.

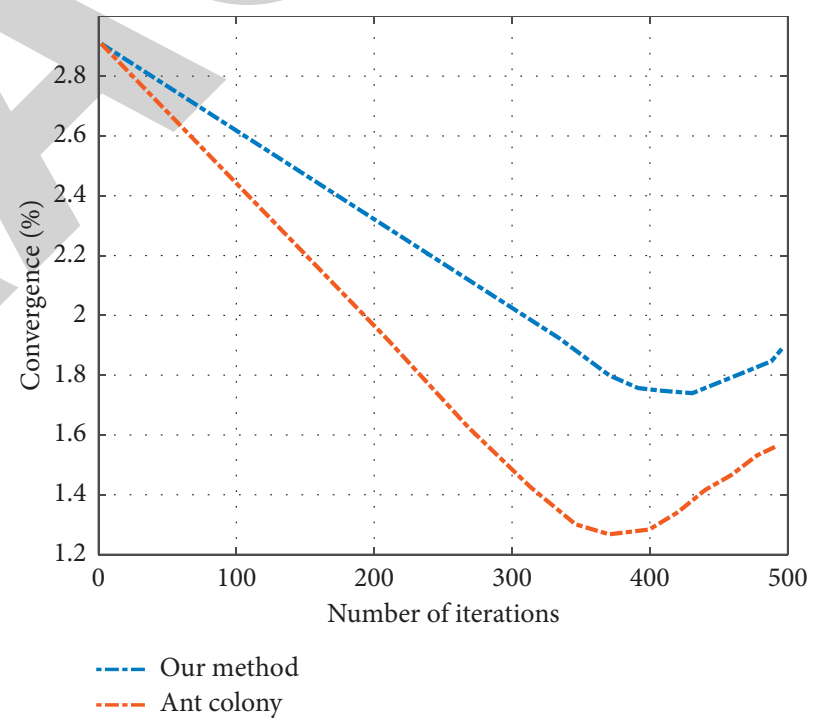

FIgURE 5: Comparison of convergence of badminton backcourt hitting route planning.

In order to further verify the superior performance of this method, the badminton backcourt stroke route planning method based on the small-world model, the badminton backcourt stroke route planning method based on ant colony optimization, and the badminton backcourt stroke route planning method based on fuzzy PID are used as comparison methods to test the convergence ability of badminton backcourt stroke route planning under different methods. The comparison results are shown in Figure 5.

The analysis of the above simulation results shows that the convergence performance of this method for badminton backcourt stroke path planning is better, which is obviously better than other methods. It shows that this method has strong adaptive planning ability for badminton backcourt 
stroke path planning and can effectively improve the reliability of badminton backcourt stroke path planning.

\section{Conclusions}

In this study, an optimized badminton backcourt stroke line planning model was constructed which is based on deep learning. Combined with the optimization control method of the badminton backcourt stroke line, the artificial intelligence learning algorithm was used to realize the badminton backcourt stroke line planning and design. The adaptive learning method of motion primitive trajectory was used for the planning and design of badminton backcourt hitting line nodes and path space. Moreover, the shortest path optimization parameter analysis model of badminton backcourt hitting line planning was constructed. Furthermore, the piecewise route optimization control method was used to design the badminton backcourt hitting line distribution grid model. The combination of the deep learning method and the intelligent planning of the badminton backcourt stroke line was carried out. Given the spatial planning matrix of the badminton backcourt stroke line, the error measurement parameters of badminton backcourt stroke line planning were obtained according to the threedimensional point cloud information of the scene, so as to realize the adaptive optimization of badminton backcourt stroke line planning and the motion path planning and design of the target position. The analysis showed that the proposed method has good adaptive optimization and control ability and strong convergence.

\section{Data Availability}

The data used to support the findings of this study are available upon request to the author.

\section{Conflicts of Interest}

The author declares no conflicts of interest.

\section{Acknowledgments}

This paper was supported by the Project of Shanxi Provincial Department of Science and Technology, Study on driving mechanism of gyro wheel by coupling human motion and hinge mechanism (2011026-2).

\section{References}

[1] Y. Yang, "Research on three-dimensional methods to psychology skills training of badminton players in college," Advanced Materials Research, vol. 267, pp. 837-842, 2011.

[2] X. Zhao and S. Li, "A biomechanical analysis of lower limb movement on the backcourt forehand clear stroke among badminton players of different levels," Applied Bionics and Biomechanics, vol. 2019, Article ID 7048345, 8 pages, 2019.

[3] B. Zhang, L. Feng, and W. Q. Jiang, "Mixed doubles match technical and tactical analysis of world badminton champion based on mathematical statistics," Advances in Physical Education, vol. 3, no. 4, pp. 154-157, 2013.
[4] X. Zhao and Y. Gu, "Single leg landing movement differences between male and female badminton players after overhead stroke in the backhand-side court," Human Movement Science, vol. 66, pp. 142-148, 2019.

[5] F. Josue, M. F. Abdullah, I. Zulkapri et al., "Movement pattern in term of court coverage among top international male and female badminton players during BWF World Championships 2013," Jurnal Sains Sukan \& Pendidikan Jasmani, vol. 9, no. 1, pp. 9-14, 2020.

[6] M.-H. Hung, C.-Y. Chang, K.-C. Lin, C.-L. Hung, and C.-S. Ho, "The applications of landing strategies in badminton footwork training on a backhand side lateral jump smash," Journal of Human Kinetics, vol. 73, no. 1, pp. 19-31, 2020.

[7] R. L. Eason, T. L. Smith, and E. Plaisance, "Effects of proactive interference on learning the tennis backhand stroke," Perceptual and Motor Skills, vol. 68, no. 3, pp. 923-930, 1989.

[8] T. Xie, C. Zhang, Z. Zhang et al., "Utilizing active sensor nodes in smart environments for optimal communication coverage," IEEE Access, vol. 7, pp. 11338-11348, 2018.

[9] B. Yao and N. Liang, "A smart position optimization scheme for badminton doubles based on human-computer interactive training in wireless sensor networks," EURASIP Journal on Wireless Communications and Networking, vol. 2020, no. 1, pp. 1-16, Article ID 233, 2020.

[10] M. Marei, "Employment methodology of proposed specific agility tests as a basis for planning and controlling specific physical preparation of badminton juniors experimental guiding study for coaches," Assiut Journal of Sport Science and Arts, vol. 216, no. 2, pp. 141-178, 2016.

[11] C. M. Chan and J. S. Rossmann, "Badminton shuttlecock aerodynamics: synthesizing experiment and theory," Sports Engineering, vol. 15, no. 2, pp. 61-71, 2012.

[12] Z. Hui, Y. Lijuan, and H. Jinju, "Computer-aided game analysis of net sports in preparation of Chinese teams for beijing olympics," International Journal of Computer Science in Sport (International Association of Computer Science in Sport), vol. 9, no. 3, 2010.

[13] P. Iserbyt, P. Ward, and W. Li, "Effects of improved content knowledge on pedagogical content knowledge and student performance in physical education," Physical Education and Sport Pedagogy, vol. 22, no. 1, pp. 71-88, 2017.

[14] C. Zhang, T. Xie, K. Yang et al., "Positioning optimisation based on particle quality prediction in wireless sensor networks," IET Networks, vol. 8, no. 2, pp. 107-113, 2019. 